\title{
Natural image noise removal using nonlocal means and hidden Markov models in transform domain
}

\begin{abstract}
Nonlocal means (NLM) which utilizes the self-similarity is considered as one of the most popular denoising techniques. Although NLM can attain significant performance, it shows a few loopholes, such as its computational complexity when it comes to similarity measurements, and the small number of sufficient candidates that use to choose the target patches which have complicated textures. In this paper, the use of clustering based on moment invariants and the hidden Markov model (HMM) is proposed to achieve preclassification and thus capture the dependency between additive white Gaussian noise pixel and its neighbors on the wavelet transform. The HMM also allows hidden states to connect to one another to capture the dependencies among coefficients in the transform domain. In the practical part, the experimental results present that the proposed algorithm has the ability to show denoised images better than the results of state-of-the-art denoising methods both objectively in peak signal-to-noise ratio and structural similarity and subjectively using visual results, especially when the noise level is high.
\end{abstract}

Keyword: Gaussian noise; Nonlocal means; Hidden Markov model; Spatial filter; Noise removal; Classification clustering. 